\title{
Curved Space (Matrix) Membranes
}

\author{
Jens Hoppe \\ Department of Mathematics, Royal Institute of Technology, 10044 Stockholm
}

\begin{abstract}
Hamiltonian formulations of M-branes moving in curved backgrounds are given.
\end{abstract}

As is well known (see e.g. [1], [2]), varying the volume

$$
\begin{gathered}
\text { Vol } \mathcal{M}=S\left[x^{\mu}\right]=\int d^{M+1} \varphi \sqrt{G} \\
G=\left|\operatorname{det}\left(\frac{\partial x^{\mu}}{\partial \varphi^{\alpha}} \frac{\partial x^{\nu}}{\partial \varphi^{\beta}} \eta_{\mu \nu}(x)\right)\right|
\end{gathered}
$$

of an $M+1$ dimensional (time-like) manifold $\mathcal{M}$ embedded in a Lorentzian manifold $\mathcal{L}$ one obtains

$$
\begin{array}{r}
\frac{1}{\sqrt{G}} \partial_{\alpha}\left(\sqrt{G} G^{\alpha \beta} \partial_{\beta} x^{\mu}\right)+G^{\alpha \beta} \partial_{\alpha} x^{\nu} \partial_{\beta} x^{\lambda} \Gamma_{\nu \lambda}^{\mu}(x)=0 \\
\mu=0,1, \ldots N
\end{array}
$$

as equations of motion. Assuming that $\mathcal{L}=\mathbb{R} \times \mathcal{N}$, and choosing [3]

$$
\begin{array}{r}
\varphi^{0}=x^{0}=: t, G_{0 b}=0 \\
(b=1, \ldots M),
\end{array}
$$

referred to as $\mathrm{O}$ (rdinary)T(ime) Orthonormal Gauge, (3) $\mu=0$ implies that

$$
\rho:=\left(\frac{\operatorname{det}\left(\partial_{a} x^{i} \partial_{b} x^{j} \eta_{i j}(x)\right)}{1-\dot{x}^{i} \dot{x}^{j} \eta_{i j}(x)}\right)^{1 / 2}
$$


is time-independent, and (3) $\mu=i . . N$ then takes the form

$$
\ddot{x}^{i}+\dot{x}^{j} \dot{x}^{k} \Gamma_{j k}^{i}(x)=\frac{1}{\rho} \partial_{a}\left(\frac{g}{\rho} g^{a b} \partial_{b} x^{i}\right)+\frac{g}{\rho^{2}} g^{a b} \partial_{a} x^{j} \partial_{b} x^{k} \Gamma_{j k}^{i}(x) .
$$

As shown below, (5) -together with (3) and (4), are Hamiltonian with respect to

$$
\begin{gathered}
H=\int d^{M} \varphi \sqrt{p_{i} \eta^{i j}(x) p_{j}+\operatorname{det}\left(\partial_{a} x^{i} \partial_{b} x^{j} \eta_{i j}(x)\right)}=: \int d^{M} \varphi \mathcal{H}, \\
p_{i} \partial_{a} x^{i}=0(a=1 \ldots M)
\end{gathered}
$$

and 1 the Hamiltonian density $\mathcal{H}=\sqrt{p^{2}+g}$ is time independent (and equal to $\rho$ ), just as in the case of flat backgrounds [4], [5]. The equations of motion following from ([6) are

$$
\begin{array}{r}
\dot{x}^{i}=\frac{\delta H}{\delta p_{i}}=\frac{\eta^{i j}(x)}{\mathcal{H}} p_{j} \\
\dot{p}_{i}=\frac{-\delta H}{\delta x^{i}}=\frac{-1}{2 \mathcal{H}} p_{j} p_{k} \partial_{i} \eta^{j k}-\frac{1}{2 \mathcal{H}} g g^{a b} \partial_{a} x^{j} \partial_{b} x^{k} \partial_{i} \eta_{j k} \\
+\partial_{a}\left(g g^{a b} \eta_{i j} \frac{\partial_{b} x^{j}}{\mathcal{H}}\right) .
\end{array}
$$

Using (e.g.)

$$
\begin{array}{r}
\partial_{c} x^{i} \partial_{a}\left(g g^{a b} \eta_{i j} \frac{\partial_{b} x^{j}}{\mathcal{H}}\right)=g g^{a b} \eta_{i j} \partial_{c} x^{i} \partial_{b} x^{j} \partial_{a}\left(\frac{1}{\mathcal{H}}\right) \\
+\frac{1}{\mathcal{H}} \partial_{a}\left(g g^{a b} \eta_{i j} \partial_{c} x^{i} \partial_{b} x^{j}\right)-\frac{1}{\mathcal{H}} g g^{a b} \eta_{i j} \partial_{b} x^{j} \partial_{a c}^{2} x^{i} \\
=g \partial_{c}\left(\frac{1}{\mathcal{H}}\right)+\frac{1}{\mathcal{H}} \partial_{c} g-\frac{1}{2 \mathcal{H}} \partial_{c} g+\frac{1}{2 \mathcal{H}} g g^{a b} \partial_{a} x^{j} \partial_{b} x^{k} \partial_{c} \eta_{j k}
\end{array}
$$

one first shows that

\footnotetext{
${ }^{1}$ similar results were, as far as I know, also perceived by V. Moncrief, who tried to generalize [4, 5] to curved backgrounds, one or two years ago.
} 


$$
\partial_{t}\left(p_{i} \partial_{c} x^{i}\right)=\ldots=\frac{1}{2 \mathcal{H}} \partial_{c}\left(p_{i} \eta^{i j} p_{j}+g\right)+\left(p^{2}+g\right) \partial_{c} \frac{1}{\mathcal{H}}=0
$$

and then, using (7) (three times),

$$
\dot{\mathcal{H}}=0 \text {. }
$$

Note that in the case of membranes,

$$
g / \rho^{2}=-\frac{1}{2}\left\{x^{i}, x^{k}\right\} \eta_{k j}\left\{x^{j}, x^{l}\right\} \eta_{l i}
$$

if

$$
\{f, h\}:=\frac{\in^{a b}}{\rho} \partial_{a} f \partial_{b} h,
$$

and that, due to

$$
g g^{a b}=\epsilon^{a a^{\prime}} \epsilon^{b b^{\prime}} g_{a^{\prime} b^{\prime}}
$$

when $M=2$, the r.h.s. of (5) may be written as

$$
\left\{\eta_{j k}\left\{x^{i}, x^{k}\right\}, x^{j}\right\}+\left\{x^{j}, x^{l}\right\}\left\{x^{k}, x^{m}\right\} \eta_{l m} \Gamma_{j k}^{i},
$$

hence allowing a matrix model approximation of (15). However, as in the case of flat backgrounds [3], due to the constraints (77) and the square root in (6), (especially with respect to quantization), it can be advantageous to use lightcone coordinates and (assuming $\mathcal{M}=\mathbb{R}^{1,1} \times \tilde{\mathcal{N}}$ ) choose the $\mathrm{L}$ (ight)C(one) ONG (cp [3] for $\left.\tilde{\mathcal{N}}=\mathbb{R}^{d}\right)$

$$
\begin{array}{r}
\varphi^{0}=\frac{x^{0}+x^{d+1}}{2}, \\
G_{0 b}=0(b=1 \ldots M) .
\end{array}
$$

One again obtains (5), but now with

$$
i j=1 \ldots N-1=: d
$$

and the integrability of 


$$
0=G_{0 b}=\partial_{b} \zeta-\eta_{i j} \dot{x}^{j}\left(\partial_{b} x^{i}\right),
$$

allowing to determine $\zeta=x^{0}-x^{d+1}$, implies

$$
\partial_{a}\left(\eta_{i j} \dot{x}^{j}\right) \partial_{b} x^{i}-\partial_{b}\left(\eta_{i j} \dot{x}^{j}\right) \partial_{a} x^{i}=0
$$

instead of (7). With

$$
\rho=\left(\frac{\operatorname{det}\left(\partial_{a} x^{i} \partial_{b} x^{j} \eta_{i j}(x)\right)}{2 \dot{\zeta}-\dot{x}^{i} \dot{x}^{j} \eta_{i j}}\right)^{1 / 2}
$$

being again a time independent, non-dynamical density, a light-cone Hamiltonian description is then given by

$$
\begin{gathered}
H=\frac{1}{2} \int \frac{d^{M} \varphi}{\rho}\left(p_{i} \eta^{i j} p_{j}+g\right), \\
\partial_{a} p_{i} \partial_{b} x^{i}-\partial_{b} p_{i} \partial_{a} x^{i}=0 .
\end{gathered}
$$

In particular, for $M=2$ (see [6],[7] for some related results)

$$
\begin{gathered}
H=\frac{1}{2} \int d^{2} \varphi \rho\left(\frac{p_{i}}{\rho} \eta^{i j} \frac{p_{j}}{\rho}-\frac{1}{2}\left\{x^{i}, x^{k}\right\} \eta_{k j}\left\{x^{j} x^{l}\right\} \eta_{l i}\right) \\
\left\{p_{i}, x^{i}\right\}=0
\end{gathered}
$$

obviously leading to a matrix model with

$$
\begin{array}{r}
H \sim \operatorname{Tr}\left(\mathrm{P}_{\mathrm{i}} \eta^{\mathrm{ij}}(\mathrm{X}) \mathrm{P}_{\mathrm{j}}+\frac{1}{2}\left[\mathrm{X}^{\mathrm{i}}, \mathrm{X}^{\mathrm{k}}\right] \eta_{\mathrm{kj}}(\mathrm{X}) \eta_{\mathrm{li}}(\mathrm{X})\left[\mathrm{X}^{\mathrm{j}}, \mathrm{X}^{\mathrm{l}}\right]\right) \\
\sum_{i=1}^{d}\left[P_{i}, X^{i}\right]=0 .
\end{array}
$$

\section{Acknowledgements}

I would like to thank Joakim Arnlind and Martin Bordemann for discussions, and Jan Plefka for bringing reference [6] to my attention when discussing together after my seminar at the Albert Einstein Institute. 


\section{References}

[1] L.P. Eisenhart; (Non-)Riemannian Geometry, Princeton University Press 1927

[2] M. Bordemann, J. Hoppe; "The Dynamics of Relativistic Membranes II" Phys. Lett. B325 (1994) 359 (hep-th/9309025)

[3] J. Hoppe; "Quantum theory of a massless relativistic surface and a two-dimensional bound state problem" Ph.D. thesis, MIT 1982 http://dspace.mit.edu/handle/1721.1/15717

[4] J. Hoppe; "Canonical 3+1 Description of Relativistic Membranes", hep-th/9407103

[5] V. Moncrief; "Can one ADM quantize relativistic bosonic strings and membranes?" Gen. Rel. Grav. (2006) 38(4):561

[6] Jongwook Kim, Nakwoo Kim, Jeong-Hyuck Park, Jan Plefka "M-theory on pp-waves with a holomorphic superpotential and its membrane and matrix descriptions" JHEP 0808:089,2008 (arXiv:0804.3349), and references therein.

[7] H. Shimada; " $\beta$-deformation for matrix model of M-theory" Nucl. Phys. B813 (2009) 283-314 (arXiv:0804.3236) 